Stochastic Hydrol. Hydraul. 4 (1990) 193-208

Stochastic Hydrology and Hydraulics

(C) Springer-Verlag 1990

\title{
Parameter identification in tidal models with uncertain boundary conditions
}

\author{
P. G. J. ten Brummelhuis \\ University of Twente, Dep. of Mathematics, P.O. Box 217, 7500 AE Enschede, The \\ Netherlands
}

\section{A. W. Heemink}

Tidal Waters Division, P.O. Box 20907, 2500 EX The Hague, The Netherlands

\begin{abstract}
In this paper a parameter estimation algorithm is developed to estimate uncertain parameters in two dimensional shallow water flow models. Since in practice the open boundary conditions of these models are usually not known accurately, the uncertainty of these boundary conditions has to be taken into account to prevent that boundary errors are interpreted by the estimation procedure as parameter fluctuations. Therefore the open boundary conditions are embedded into a stochastic environment and a constant gain extended Kalman filter is employed to identify the state of the system. Defining a error functional that measures the differences between the filtered state of the system and the measurements, a quasi Newton method is employed to determine the minimum of this functional. To reduce the computational burden, the gradient of the criterium that is required using the quasi Newton method is determined by solving the adjoint system.
\end{abstract}

Key words: Stochastic tidal modeling, parameter identification, model calibration.

\section{Introduction}

In trying to understand processes that are associated with water movements one can make use of a number of techniques. The process can be observed or it can be simulated numerically. The applications can vary from a study the spread of pollutants in shallow waters to the forecast of storm surges in coastal areas. Before a numerical model can be used as an instrument to predict processes accurately, it has to be ascertained that the model is reliable. This can be performed by adjusting the model such that the model outcome represents a series of observations as good as possible. The adjustment of the model implies that some quantities appearing in the formal equations describing the system dynamics must be specified. With respect to these quantities one may think of the geometry, of boundary conditions, of the location of the pollution sources and their intensity or of some empirical parameters. Usually this is called the calibration of the numerical model. In this paper we will give no extensive treatment of the total process of developing a model, but we will describe a procedure to perform the calibration automatically. Up till now there was no such procedure. This does not mean that, based on an extensive experience, the calibration could not be done accurately by hand. Moreover, it is not at forehand guaranteed that the calibration results of a systematic procedure will be significantly better. However, calibrating a model by hand is very time consuming. Furthermore, using an automatic calibration procedure models can also be developed by less experienced users and finally the results of different calibration sessions can be compared. This is important since the available measurement information is usually too small 


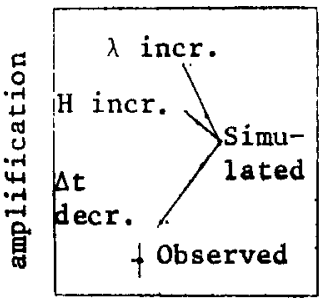

lag

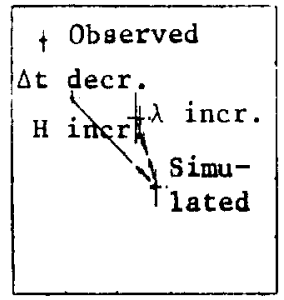

$\operatorname{lag}$

Figure 1. An amplitude-phase portrait of the $M_{2}$ and $M_{4}$ tidal constituent

to determine a unique set of values for all the quantities that are not exactly known. One of the major difficulties in tuning a model by hand is the lack of insight into the complex behaviour of the model. This is best illustrated by a short description of how the calibration is often accomplished (Langerak, personal communication). To a large extent, the results of a tidal model can be expressed in terms of harmonic constants. The calibration of a model can therefore be performed by trying to generate a model solution in the data observation points whose harmonic constants are equal to the constants of the observed data in this point: for each important tidal constituent the amplitude and phase must be the same (or the difference has to be minimized). This is graphically illustrated by a phase-amplitude portrait, see figure 1 . Because of the non-linearity of the dynamics, the different tidal constituents can not be treated separately. Moreover this interaction makes it hard to find a unique set of parameter values that globally minimize the difference between the observed data set and the model outcome.

We will describe in this paper a parameter estimation procedure to determine a number of system parameters in the presence of uncertain boundary conditions. In case the system dynamics are described by the 1-D shallow water equations, in the literature some examples are described concerning the estimation of system parameters. Budgell (1981) estimates friction coefficients in channel branches off-line by maximizing a log likelihood function in combination with state estimation using a Kalman filter. Chao-lin Chiu (1978) estimates bottom friction coefficients in steady state channel flow on-line using an extended Kalman filter. The last application to be mentioned here is a method where the parameters are simultaneously estimated with the state in case of unsteady flow in branched estuaries using extended Kalman filtering (ten Brummelhuis et al., 1988; Heemink and ten Brummelhuis, 1989; Heemink, 1988). For the case the dynamics are described by the 2-D shallow water equations, the techniques just described are not applicable because of computational limitations (both computational time and memory requirements). Therefore we choose to develop an iterative off-line parameter estimation procedure. As a consequence of the fact that the procedure will also be applied in cases where the open boundary conditions are uncertain, we combine the parameter estimation with state estimation.

At the end of this introduction we give an overview of the contents of this paper. In section 2 the shallow water equations are formulated in a stochastic way to account for uncertainties in the open boundary conditions. The numerical representation of this system is treated in section 3. The total estimation procedure is described in section 4 (the state estimation) and 5 (the parameter estimation). After the presentation of some theoretical aspects of the method, results are commented in the sections 6 and 7, where a distinction is made between the cases with and without uncertainties in the prescription of the open boundary conditions. Finally, section 8 contains some conclusions. 


\section{Shallow water equations}

The two-dimensional non-linear shallow water equations to describe the shallow water flow in coastal waters are (Dronkers, 1964);

$$
\begin{aligned}
& \frac{\partial u}{\partial t}+u \frac{\partial u}{\partial x}+v \frac{\partial u}{\partial y}+g \frac{\partial h}{\partial x}-f v+\lambda(u, v) \frac{u}{D+h}=\frac{\tau_{x}}{D+h}-\frac{1}{\rho_{w}} \frac{\partial p_{a}}{\partial x} \\
& \frac{\partial v}{\partial t}+u \frac{\partial v}{\partial x}+v \frac{\partial v}{\partial y}+g \frac{\partial h}{\partial y}+f u+\lambda(u, v) \frac{v}{D+h}=\frac{\tau_{y}}{D+h}-\frac{1}{\rho_{w}} \frac{\partial p_{a}}{\partial y} \\
& \frac{\partial h}{\partial t}+\frac{\partial[u(D+h)]}{\partial x}+\frac{\partial[v(D+h)]}{\partial y}=0
\end{aligned}
$$

where:

$$
\begin{array}{ll}
h & =\text { water-level } \\
u, v & =\text { water velocities in respectively the } \mathrm{x} \text { and } \mathrm{y} \text {-direction } \\
D & =\text { depth of the water } \\
f & =\text { Coriolis parameter } \\
\lambda & =\text { bottom friction coefficient } \\
\tau_{x}, \tau_{y} & =\gamma V^{2} \cos \psi, \gamma V^{2} \sin \psi=\text { wind stress in respectively the } x \text { and } y \text {-direction where: } \gamma \\
& =\text { wind friction coefficient, } V=\text { wind speed, } \psi=\text { wind direction } \\
\rho_{w} \quad & =\text { density of water } \\
p_{a} \quad & =\text { atmospheric pressure } \\
g & =\text { acceleration of gravity }
\end{array}
$$

In case the water is rather deep, the non-linearities in the equations (1)-(3) are small and it is possible to approximate these equations by the linear ones:

$$
\begin{aligned}
& \frac{\partial u}{\partial t}+g \frac{\partial h}{\partial x}-f v+\lambda \frac{u}{D}=\frac{\tau_{x}}{D}-\frac{1}{\rho_{w}} \frac{\partial p_{a}}{\partial x} \\
& \frac{\partial v}{\partial t}+g \frac{\partial h}{\partial y}+f u+\lambda \frac{v}{D}=\frac{\tau_{y}}{D}-\frac{1}{\rho_{w}} \frac{\partial p_{a}}{\partial y} \\
& \frac{\partial h}{\partial t}+\frac{\partial(u D)}{\partial x}+\frac{\partial(v D)}{\partial y}=0
\end{aligned}
$$

The wave motion is completely described by the non-linear equations (1)-(3) or the linear ones (4)-(6) provided that initial values and the closed and open boundary conditions are given. At a closed boundary the velocity normal to the boundary is zero:

$v_{\perp}=0$

There is a considerable uncertainty associated with open boundary conditions. In this paper we represent this uncertainty in a stochastic sense, and describe it formally by:

$h=f(t)+H \quad ; \quad d H=-\alpha H d t+\sigma_{b} d W$

Here we note that since $\sigma_{b}$ is constant the stochastic differential equations (8) and (9) are the same in the Ito and the Stratonowitz sense and can be manipulated by formal rules (Jazwinski, 1970). The statistics of the Brownian motion process $W$ are assumed to be: 
196

$E\{d W(x, t)\}=0 \quad ; \quad E\left\{d W\left(x_{1}, t\right) d W\left(x_{2}, t\right)\right\}=e^{-\delta\left|x_{1}-x_{2}\right|} d t$

As a result the statistics of the coloured noise process $H$ are:

$E\{\dot{H}(x, t)\}=0 \quad ; \quad E\left\{H\left(x_{1}, t_{1}\right) H\left(x_{2}, t_{2}\right)\right\}=\left[\sigma_{b}^{2} e^{-\alpha\left|t_{2}-t_{1}\right|-\delta\left|x_{1}-x_{2}\right|}\right] / 2 \alpha$

The colourdness of the stochastic process $H$ is argued by several reasons: One of the common ways to describe open boundary conditions like (8)-(9) is to use measurements at some points at the boundary and to interpolate them in between. As a result errors are introduced. Because we are dealing with a tidal motion problem, the water levels can be expressed by a limited number of harmonic constituents which determine the tide to a large extent, and a great number of additional harmonic components which are of minor importance. Consequently, in the spectrum of the errors at the open boundary condition some (low frequent) harmonic constituents will be dominant while the remaining part can be approximated by a white noise process. The second form of errors to be introduced at the open boundaries originate outside the considered domain when a severe storm creates a surge which propagates across the open boundary into the model and which disturbes the constituents of very low frequencies.

In the non-linear case it has to be assumed, to ensure the well posedness of the problem, that at inflow boundaries the velocity parallel to the open boundary is zero:

$v_{\mid 1}=0$

\section{Numerical approximation}

The shallow water equations are approximated by using an Alternating Direction Implicit (ADI) method. The numerical scheme is developed by Stelling (1983) and is based on the well-known work of Leendertse (1967). It is unconditionally stable and lacks artificial viscosity. Defining a space-staggered grid $G_{1}$ (see figure 2 ), the finite difference equations to approximate the homogeneous part of the linear equations are:

step 1:

explicit:

$$
\begin{aligned}
v_{m, n+1}^{k+\frac{1}{2}}= & \left(v_{m, n+1}^{k}-g \frac{\Delta t}{2 \Delta y}\left(h_{m, n+1}^{k}-h_{m, n}^{k}\right)+\frac{1}{8} \Delta t f\left(u_{m-1, n+1}^{k}+u_{m-1, n}^{k}+u_{m+1, n+1}^{k}\right.\right. \\
& \left.\left.+u_{m+1, n}^{k}\right)\right) /\left(1+\frac{\Delta t \lambda_{m, n+1}}{D_{m-1, n+1}+D_{m+1, n+1}}\right)
\end{aligned}
$$

implicit:

$$
\begin{aligned}
& \left(h_{m, n}^{k+\frac{1}{2}}-h_{m, n}^{k}\right)+\frac{\Delta t}{4 \Delta x}\left[\left(D_{m+1, n+1}+D_{m+1, n-1}\right) u_{m+1, n}^{k+\frac{1}{2}}-\left(D_{m-1, n+1}+D_{m-1, n-1}\right) u_{m-1, n}^{k+\frac{1}{2}}\right] \\
& +\frac{\Delta t}{4 \Delta y}\left[\left(D_{m+1, n+1}+D_{m-1, n+1}\right) v_{m, n+1}^{k}-\left(D_{m+1, n-1}+D_{m-1, n-1}\right) v_{m, n-1}^{k}\right]=0 \\
& \left(u_{m+1, n}^{k+\frac{1}{2}}-u_{m+1, n}^{k}\right)+g \frac{\Delta t}{2 \Delta y}\left(h_{m+1, n}^{k+\frac{1}{2}}-h_{m, n}^{k+\frac{1}{2}}\right)-\frac{1}{8} \Delta t f\left(v_{m, n+1}^{k+\frac{1}{2}}+v_{m+1, n+1}^{k+\frac{1}{2}}\right.
\end{aligned}
$$



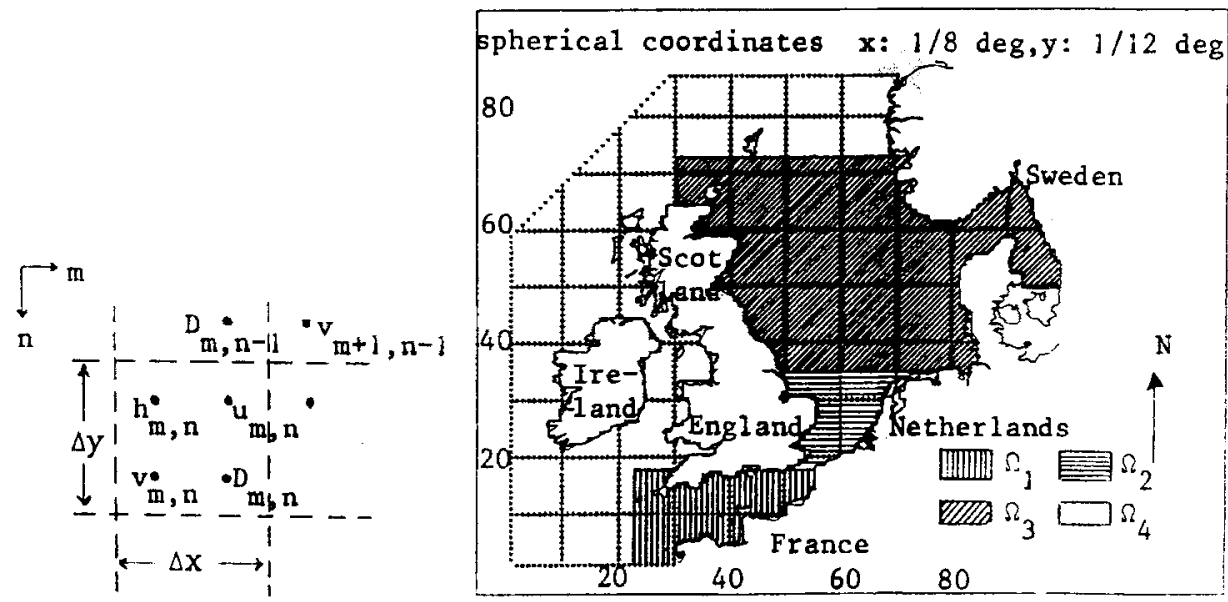

Figure 2

Figure 3

Figure 2. The space-staggered grid $G_{1}$

Figure 3. The European continental shelf model

$$
+v_{m+1, n-1}^{k+\frac{1}{2}} \underset{+v_{m+1, n-1}^{k+\frac{1}{2}}}{+\lambda_{m+1, n+1}+D_{m+1, n-1}}+\frac{\lambda_{m+\frac{1}{2}}^{k}}{D_{m+1, n}}=0
$$

step 2:

explicit:

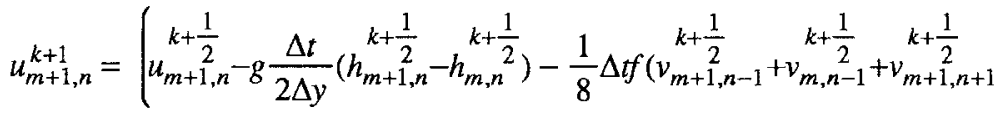

$$
\begin{aligned}
& \left.+v_{m, n+1}^{k+\frac{1}{2}}\right) /\left(1+\frac{\Delta t \lambda_{m+1, n}}{D_{m+1, n+1}+D_{m+1, n-1}}\right)
\end{aligned}
$$

implicit:

$$
\begin{aligned}
& \left(h_{m, n}^{k+1}-h_{m, n}^{k+\frac{1}{2}}\right)+\frac{\Delta t}{4 \Delta x}\left(\left(D_{m+1, n+1}+D_{m+1, n-1}\right) u_{m+1, n}^{k+\frac{1}{2}}-\left(D_{m-1, n+1}+D_{m-1, n-1}\right) u_{m-1, n}^{k+\frac{1}{2}}\right) \\
& +\frac{\Delta t}{4 \Delta y}\left(\left(D_{m+1, n+1}+D_{m-1, n+1}\right) v_{m, n+1}^{k+1}-\left(D_{m+1, n-1}+D_{m-1, n-1}\right) v_{m, n-1}^{k+1}\right)=0 \\
& \left(v_{m, n+1}^{k+1}-v_{m, n+1}^{k+\frac{1}{2}}\right)+g \frac{\Delta t}{2 \Delta y}\left(h_{m, n+1}^{k+1}-h_{m, n}^{k+1}\right)+\frac{1}{8} \Delta t f\left(u_{m-1, n}^{k+1}+u_{m-1, n+1}^{k+1}+u_{m+1, n+1}^{k+1}\right. \\
& \left.+u_{m+1, n+1}^{k+1}\right)+\frac{\lambda_{m, n+1} \Delta t v_{m, n+1}^{k+1}}{D_{m-1, n+1}+D_{m+1, n+1}}=0
\end{aligned}
$$

For the details such as the numerical boundary treatment and the non-linear case we refer to Stelling (1983).

The stochastic differential equation (9) is approximated using the Euler scheme: 

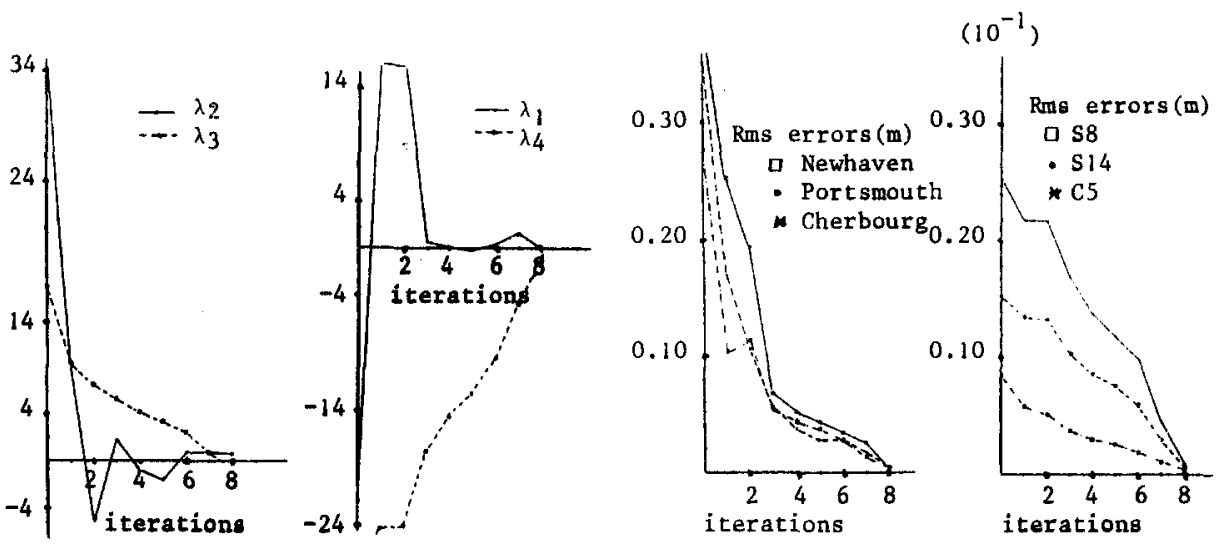

Figure 4

Figure 5

Figure 4. The estimated coefficients $\lambda_{i}$. In the dynamical equations of this model the bottom friction coefficient is represented by the Chezy formula: $\left(g \sqrt{u^{2}+v^{2}}\right) / \lambda_{m, n}^{2}$

Figure 5. The r.m.s. in the subdomains $\Omega_{1}$ and $\Omega_{4}$

$H_{m}^{k+1}=(1-\alpha \Delta t) H_{m}^{k}+\sigma_{b}\left(W_{m}^{k+1}-W_{m}^{k}\right)$

The meteorological input processes $\tau_{x}, \tau_{y}$ and $p_{a}$ are available on a grid $G_{2}$. Since these processes are less spatially variable than the underlying water wave process, $G_{2}$ is much coarser than the grid $G_{1}$ and therefore, interpolation is necessary to obtain these quantities in the grid points of $G_{1}$.

\section{Kalman filtering}

A state-space representation of the linear model is obtained by defining an $n$-vector $\underline{X}^{k}$ that contains the water level and velocities in all the grid points of $G_{1}$. The finite difference scheme can now be rewritten as a deterministic system:

$A \underline{X}^{k+\frac{1}{2}}=B \underline{X}^{k}+R \underline{u}^{k+\frac{1}{2}} \quad ; \quad C \underline{X}^{k+1}=D \underline{X}^{k+\frac{1}{2}}+R \underline{u}^{k+1}$

Here $A, B, C$ and $D$ are coefficient matrices representing the ADI scheme and $u^{k}$ is the meteorological input given on the grid $G_{2}$. The matrix $R$ represents a sequence of linear operations to interpolate the meteorological input at the grid points of $G_{1}$. Similarly, we define the $q$-vector $q^{k}$ to contain the coloured noise processes introduced in the open boundary conditions. The stochastic dynamic model can now be described by:

$$
\left[\begin{array}{cc}
C & 0 \\
0 & I
\end{array}\right]\left[\begin{array}{l}
\underline{X}^{k+1} \\
\underline{q}^{k+1}
\end{array}\right]=\left[\begin{array}{cc}
D A^{-1} B & E \\
0 & 1-\alpha \Delta t
\end{array}\right]\left[\begin{array}{c}
\underline{X}^{k} \\
\bar{q}^{k}
\end{array}\right]+\left[\begin{array}{c}
D A^{-1} R \\
0
\end{array}\right] \underline{u}^{k+\frac{1}{2}}+\left[\begin{array}{c}
R \\
0
\end{array}\right] \underline{u}^{k+1}+\left[\begin{array}{c}
0 \\
\underline{W}_{b}^{k}
\end{array}\right]
$$

Here $E$ is a coefficient matrix representing the open boundary treatment and $W_{b}^{k}$ contains the increments of the Brownian motion processes introduced in the discretised open 
boundary conditions. The covariance matrix of $W_{b}^{k}$ can be derived easily from equations (10)-(11). Since the model is of the hyperbolic type, the effect of the initial condition, which is often poorly known, vanishes after a limited period of time. Equation (24) can be rewritten as:

$\tilde{A} \underline{Y}^{k+1}=\tilde{B} \underline{Y}^{k}+\underline{u}^{k+1}+\underline{\tilde{W}}^{k+1}$

while $\tilde{Q}$ is defined as covariance matrix of $\bar{W}^{k}$.

In the non-linear case the stochastic system representation of the model becomes:

$\tilde{A} \underline{Y}^{k+1}=\underline{\tilde{b}}\left(\underline{Y}^{k}, \underline{u}^{k+1}\right)+\underline{\tilde{W}}^{k+1}$

Assuming that measurements of the water level are available at $m$ grid points of $G_{1}$, the observation equation can be derived easily:

$\underline{Z}^{k}=M \underline{Y}^{k}+\underline{V}^{k}$

where the m-vector $\underline{Z}^{k}$ contains the measurements taken at time $k \Delta t$ and $\underline{V}^{k}$ is the white measurement noise with covariance matrix $S . V^{k}$ and $\underline{W}^{k}$ are assumed to be mutually independent. In practice, we may assume that the measurement errors at different locations are mutually independent and have equal variance, so $S$ is diagonal with elements $\sigma^{2}$. If necessary, equation (27) can be modified to account for the fact that not all measurements are available at grid points.

It is desired to combine the measurements taken from the actual system and modeled by relation (27) with the information provided by the system model (25) or (26) to obtain an estimate of the system state $\underline{Y}^{k}$. Let us first consider the linear case. If $\hat{Y}(k \mid l)$ is defined as the least squares estimate of the state $\underline{Y}^{k}$ using the measurements $\underline{Z}^{1}, \underline{Z}^{2}, \ldots, \underline{Z}^{l}$, $l \leq k$, the recursive filter equations to obtain this quantity can be summerized as follows (Heemink, 1988):

$\bar{A} \underline{\hat{Y}}(k+1 \mid k)=\hat{B} \underline{\hat{Y}}(k \mid k)+\underline{u}^{k+1}$

$\hat{\hat{Y}}(k+1 \mid k+1)=\underline{\hat{Y}}(k+1 \mid k)+K\left[\underline{Z}^{k+1}-M \underline{\hat{Y}}(k+1 \mid k)\right]$

where the steady state Kalman filter gain $K$ can be determined by the Chandrasekhartype equations to exploit the fact that in our case the dimension $q$ of the system noise is very small compared to the dimension $n$ of the state vector (Morf et al., 1974).

Let us now consider the non-linear model (26). The linearized Kalman filter can in this case be summarized as follows:

$\tilde{A} \hat{Y}(k+1 \mid k)=\tilde{b}\left(\underline{Y}(k \mid k), \underline{u}^{k+1}\right)$

$\hat{\underline{Y}}(k+1 \mid k+1)=\hat{Y}(k+1 \mid k)+K^{k+1}\left[\underline{Z}^{k+1}-M \underline{Y}(k+1 \mid k)\right]$

Here the time-varying Kalman filter gain $K^{k+1}$ is determined by linearizing $\underline{b}$ about a reference trajectory and by using the filter equations for linear time-varying systems (Jazwinski, 1970). In our case the time-variations of $K^{k+1}$ are only caused by the nonlinearities of $\tilde{b}$. However, if the water is rather deep, these non-linearities are relatively small and, consequently, the time-variations of $K^{k+1}$ are small too. Moreover, since deterministic boundary conditions usually provide a reasonable description of the tidal elevation, the correction produced by the Kalman filter by means of equation (31) is small compared to the deterministic results. Therefore a relatively small error in $K^{k+1}$ hardly effects the estimates of the filter and we may expect that a constant gain extended Kalman filter produces estimates that are nearly optimal (Safonov and Athans, 1978). This filter is based on the linearization of $\underline{b}$ about an equilibrium state and, as a consequence, 
in our case this filter is time-invariant:

$$
\begin{aligned}
& \hat{A} \underline{\hat{Y}}(k+1 \mid k)=\underline{\tilde{b}}\left(\hat{Y}(k \mid k), \underline{u}^{k+1}\right) \\
& \hat{Y}(k+1 \mid k+1)=\underline{\hat{Y}}(k+1 \mid k)+K\left[\underline{Z}^{k+1}-M \underline{\hat{Y}}(k+1 \mid k)\right]
\end{aligned}
$$

where the steady state Kalman filter gain $K$ is determined by solving the filter equations that are based on the linear model (25).

\section{The estimation procedure}

By using the Kalman filter algorithm for the estimation of the states, the errors that are due to the uncertain open boundary conditions can be compensated. Because the effects of unknown parameter values are not taken into account the filter estimate will still suffer from the errors. Therefore an additional parameter estimation procedure will be applied.

In this paper we describe an off-line procedure to estimate the values of the model parameters. The essence of the parameter estimation procedure is the iterative minimization of a socalled error functional $\Gamma(p)$, where $p$ denotes the unknown parameters. $\Gamma(p)$ is a-measure of the difference between the model output and the observed data. For the measure we take:

$\Gamma \underline{p})=\sum_{k=N_{1}}^{N_{2}} \sum_{j=1}^{N_{m}}\left\{\underline{Z}_{j}^{k}-\left(M \underline{\hat{Y}}(k \mid k, \underline{p})_{j}\right\}^{2}\right.$

with:

$\underline{Z}^{k} \quad=$ measurement vector for $t=t_{k}$

$M \quad=$ measurement matrix

$N_{1}, N_{2}=$ time indices, $N_{1} \geq 0$ and $N_{2} \leq N$

$N_{m} \quad=$ the number of data observation points

Within this procedure, it is assumed that the functional $\Gamma(p)$ can be locally described by a quadratic expression of the form:

$\Gamma(\underline{p}+\underline{\delta})=\Gamma(\underline{p})+\nabla \Gamma(\underline{p})^{T} \cdot \underline{\delta}+\frac{1}{2} \underline{\delta}^{T} G(\underline{p}) \cdot \underline{\delta}$

with $G(p)$ the Hessian. Based on equation (35) a new estimate of $p$ can be found according to:

$\underline{p}^{\prime}=\underline{p}-G(\underline{p})^{-1} \cdot \nabla \Gamma(\underline{p})$

by putting $\gamma r \Gamma(p+\underline{\delta)}=\underline{0}$.

It can be proved that, if $\bar{p}$ is close enough to the real value $p^{*}$ and $G\left(p^{*}\right)$ is positive definite, the algorithm will show a second order convergence. The disadvantages of this iterative Newton method can be found by denying the above mentioned conditions: If $G\left(p^{*}\right)$ is not positive definite or the momentary estimate of $p$ is not close to $p^{*}$, the iteration process can diverge. For this reason a Quasi Newton method is usually exploited, in combination with a line-search. The inverse of the Hessian is recursively approximated by a symmetric positive definite matrix $H(p)$. An iteration step (characterized by the index $i$ ) now consists of two stages (Fletcher, 1980):

1. Find a search direction $s\left(p_{i}\right)=-H\left(p_{i}\right) \cdot \nabla \Gamma\left(p_{i}\right)$

2. Minimize $\Gamma(p)$ along this direction $\underline{\underline{s}}\left(p_{i}\right)$ :

$$
\min _{\alpha>0} \Gamma\left(\underline{p}_{i}+\bar{\alpha} \underline{\underline{s}}\left(\underline{p}_{i}\right) \delta_{0}\right) \text { with } \delta_{0} \text { constant so that } \underline{p}_{i+1}=\underline{p}_{i}+\alpha \underline{s}\left(\underline{p}_{i}\right) \delta_{0}
$$


The difference between the various Quasi Newton methods is the way in which the matrix $H(p)$ is recursively updated. In the literature a great number alternatives can be found. Here the Broyden, Fletcher, Glodfarb and Shanno (BFGS) formula is used. The update of $H(p)$ is given by

$H\left(\underline{p}_{i+1}\right)=H\left(\underline{p}_{i}\right)+\left[1+\frac{\underline{\gamma}_{i}^{T} H\left(p_{i}\right) \gamma_{i}}{\underline{\delta}_{i}^{T} \underline{\gamma}_{i}}\right] \frac{\underline{\delta}_{i} \underline{\delta}_{i}^{T}}{\underline{\delta}_{i}^{T} \underline{\gamma}_{i}}-\left[\frac{\underline{\delta}_{i} \gamma_{i}^{T} H\left(p_{i}\right)+H\left(p_{i}\right) \gamma_{i} \underline{\delta}_{i}^{T}}{\underline{\delta}_{i}^{T} \underline{\gamma}_{i}}\right]$

with $\underline{\delta}_{i}=p_{i+1}-p_{i}$ and $\underline{\gamma}_{i}=\nabla \Gamma\left(p_{i+1}\right)-\nabla \Gamma\left(p_{i}\right)$.

It can be shown that in case the functional $\Gamma(p)$ is quadratic with respect to $p$, the number of iterations required to find the minimum is equal to the dimension of $p$. However, after we implemented the algorithm in this standard form, a serio,us disadvantage appears: it is a general shortcoming of (Quasi) Newton methods that they converge to local minima that can differ substantially from the global minimum (Fletcher, 1980). Numerous "ad hoc" solutions are known to overcome this difficulty, but these are all strongly dependent on the model at hand and the form of $\Gamma(p)$. For example, one of the suggestions was to use some relaxation to determine a new search direction. After various attempts to make the alogrithm converge with nearly second order rate in all cases, it turns out that this can be achieved by normalizing the vector $s\left(p_{i}\right)$, every time it is calculated. In doing so, the search for a minimum is performed over an interval in the parameter space which has a length $\geq \delta_{0}$.

Concerning the computational requirements, the determination of the gradient $\nabla \Gamma(p)$ is the most time consuming operation. Therefore, a variational approach is applied to find $\nabla \Gamma(p)$. It requires only one evaluation of $\Gamma(p)$ and the solution of the adjoint problem. This method has been introduced by Chavent and has the great advantage that the computational cost to determine the gradient $\nabla \Gamma(p)$ is independent of the dimension of $p$ (Chavent, 1979; N'Kaoua, 1984). In short, the approach is the following: Consider the dynamic model, equations (32)-(33), and let the state $\hat{Y}(k+1 \mid k)$ depend on a general parameter vector $p: \underline{\hat{Y}}(k+1 \mid k ; p)$. In the sequel equation $(38)$ is used as the abreviation of equations (32)-(33):

$L_{1}(k ; \underline{p})(\underline{Y}(k+1 \mid k+1, \underline{p}))=L_{2}(k ; \underline{p})(\underline{Y}(k \mid k, \underline{p}))+f_{k}$

Now, $Y$ is equal to the set of functions $\underline{\underline{Y}}(k \mid k, p)$ satisfying:

$\left.<\underline{v}, L_{1}(k ; \underline{p})(\underline{\hat{Y}}(k+1 \mid k+1, \underline{p}))\right)-L_{2}(k ; \underline{p})\left(\underline{\hat{Y}}(k \mid \hat{k}, \underline{p})-f_{k}>=0 ; k=0,1, \ldots, N-1\right.$

for all possible discrete constant test function $\underline{\underline{v}}$, or even more general, satisfying:

$\sum_{k=0}^{N-1}<\xi^{k}, L_{1}(k ; \underline{p})(\underline{\hat{Y}}(k+1 \mid k+1, \underline{p}))-L_{2}(k ; \underline{p})(\underline{\hat{Y}}(k \mid k, \underline{p}))-f_{k}>=0$

or:

$$
\begin{aligned}
\sum_{k=1}^{N-1} & <\xi^{k},\left[L_{1}(k-1 ; \underline{p})-L_{2}(k ; \underline{p})\right]\left(\underline{\hat{Y}}(k \mid k, \underline{p})-\underline{f}_{k}>-\right. \\
& <\xi^{0}, L_{2}(0 ; \underline{p})(\hat{Y}(0 \mid 0, \underline{p}))-\underline{f}_{0}>+<\xi^{N}, L_{1}(N-1 ; \underline{p})(\underline{Y}(N \mid N, \underline{p}))>=0
\end{aligned}
$$

with $\xi^{k}$ a discrete test function. The left hand side of equation (40) can be added to the originally defined criterion, equation (34), that has be minimized. This leads to equation (41): 


$$
\begin{aligned}
\hat{\Gamma}(\underline{p})= & \left.\sum_{k=N_{1}}^{N_{2}} \sum_{m}^{N_{m}}\left(\underline{Z}^{k}\right)_{j}-(M \underline{\hat{Y}}(k \mid k, \underline{p}))_{j}\right\}^{2}+\sum_{k=1}^{N-1}\left\langle\xi^{k}, L(k ; \underline{p})(\underline{\hat{Y}}(k \mid k, \underline{p}))-\underline{f}_{k}\right\rangle \\
& \left.-<\xi^{0}, L_{2}(0 ; \underline{p})(\underline{\hat{Y}}(010, \underline{p}))-\underline{f}_{0}\right\rangle+<\xi^{N} L_{1}(N-1 ; \underline{p})(\underline{\hat{Y}}(n \mid n, \underline{p}))>
\end{aligned}
$$

where $L(k ; p)$ is defined as $L_{1}(k-1 ; p)-L_{2}(k ; p)$. The variation of $\hat{\Gamma}(p)$ due to an incremental change $\Delta p$ in the $l$-th component of $p$ is:

$$
\begin{aligned}
\Delta \hat{\Gamma}(\underline{p})= & \sum_{k=1}^{N-1}<\xi^{k}, \frac{\partial L}{\partial p_{l}}(\hat{\underline{Y}}(k \mid k, \underline{p})) \Delta p+L(k ; \underline{p})(\Delta \underline{\hat{Y}}(k \mid k, \underline{p}))> \\
& -<\xi^{0}, \frac{\partial L_{2}}{\partial p_{l}}(\underline{\hat{Y}}(0 \mid 0, \underline{p})) \Delta p+L_{2}(0 ; \underline{p})(\Delta \underline{\hat{Y}}(0 \mid 0, \underline{p}))> \\
& +<\xi^{N}, \frac{\partial L_{1}}{\partial p_{l}}\left(\underline{\hat{Y}}(N \mid N, \underline{p}) \Delta p+L_{1}(N ; \underline{p})(\Delta \underline{\hat{Y}}(N \mid N, \underline{p}))>\right. \\
& -2 \sum_{k=N_{1} j=1}^{N_{2}} \sum_{m}^{N_{m}} M^{T}\left[\left(\underline{Z}^{k}\right)_{j}-(M \underline{\hat{Y}}(k \mid k, \underline{p}))_{j}\right](\Delta \underline{\hat{Y}}(k \mid k, \underline{p}))_{j}
\end{aligned}
$$

Equation (42) can be reduced to an exact expression for the derivative $\frac{\partial \Gamma}{\partial p_{l}}$ :

$$
\frac{\partial \hat{\Gamma}}{\partial p_{l}}=\sum_{k=1}^{N-1}<\xi^{k}, \frac{\partial L}{\partial p_{l}}(\underline{\hat{Y}}(k \mid k, \underline{p}))>
$$

if equations (44)-(47) hold

$<\xi^{k}, L(k ; p)(\underline{\hat{Y}}(k \mid k, p))>=$

$<L^{*}(k ; p)\left(\bar{\xi}^{k}\right), \Delta \underline{\hat{Y}}(k \mid \overline{\mid} k, p)>=<-2 M^{T}\left\{\underline{Z}^{k}-M \underline{Y}(k \mid k, p)\right\}, \Delta \underline{\hat{Y}}(k \mid k, p)>$

for $N_{1} \leq k \leq N_{2}$ and all $\bar{\Delta} \hat{Y}(k \mid k, \underline{p})$ in the tangent space of $Y$ at $\underline{\hat{Y}}(\overline{k \mid k, p})$

$<L^{*}(k ; \underline{p})\left(\xi^{k}\right), \Delta \underline{\hat{Y}}(k \mid k, \underline{p})>=0 \quad$ for $0 \leq k<N_{1}$ and $N_{2}<k \leq N-1$

$<L_{2}^{*}(0 ; \underline{p})\left(\xi^{0}\right), \Delta \underline{\underline{Y}}(010, \underline{p})>=0$

$<L_{1}^{*}(N-1 ; \underline{p})\left(\xi^{N}\right), \Delta \underline{\hat{Y}}(N \mid N, \underline{p})>=0$

In equation (44) and (45) $L^{*}(k ; p)$ denotes the adjoint operator of $L(k ; p)$ which can be determined by solving the adjoint problem:

$$
\begin{aligned}
& L^{*}(k ; \underline{p})\left(\xi^{k}\right)=-2 M^{T}\left(\underline{Z}^{k}-M \underline{\hat{Y}}(k \mid k, \underline{p})\right) \quad \text { for } N_{1} \leq k \leq N_{2} \\
& L^{*}(k ; \underline{p})\left(\Delta \xi^{k}\right)=0 \quad \text { for } 1 \leq k<N_{1} \text { and } N_{2}<k \leq N-1 \\
& \Delta \underline{\hat{Y}}(010, \underline{p})=0 \\
& \xi^{N}=0
\end{aligned}
$$

The structure of the adjoint problem is rather complicated. This is mainly due to the special characteristics of the discretization that is used to represent the linear shallow water equations numerically. Here a time step is divided into different steps. Although the equations for the steps are the same, the numerical representations are different, see equations (15)-(20). In the adjoint problem, the steps are also present, but the differential 
equivalents of the difference equations are now not the same for the different steps.

\section{Parameter estimation in deterministic models}

A special case of the calibration of tidal models is to estimate parameters in the absence of uncertainties in the open boundary conditions. In terms of the system description, this corresponds to the case where $K=0$, so the filter is not employed. The example that is described here is the identification of parameters in the CSM-16 model of the European Continental Shelf. The model equations are the discretized versions of the non-linear equations (1)-(3) while the gridsize is $16 \mathrm{~km}$. At the open boundary condition the astromomical tide is prescribed. The meteorological influences are not taken into account, so the only parameters to be estimated are bottom friction coefficients. To illustrate our approach we generate measurements by running a truth model. The friction coefficients have a distributed nature. Therefore the domain $\Omega$ is divided into a number of subdomains $\Omega_{i}, i=1,2,3,4$ where the bottom friction coefficients have a prescribed spatial dependence $F_{m, n}$ :

$\lambda_{m, n}=F_{m, n}+\lambda_{i}$

for all points $(m, n)$ in $\Omega_{i}$. Here the parameter $\lambda_{i}$ has to be estimated. In the CSM-16 model, the four subdomains are quite distinguishable: the area between England and France, the southern part the North Sea, the 'open sea' area and an area connected to the open boundary, see figure 3 . The points where simulated data are generated are indicated therein by black dots. These points coincide with real data observation points. It is seen that these points are not uniformly distributed over $\Omega$. By introducing weighting factors $\alpha_{i}, \Gamma(p)$ can be determined by the sum of the averaged squares of the residuals in the subdomains $\Omega_{i}$. A simple choice would be to take these weighting factors $\alpha_{i}$ equal to the reciproke of the number of data observation points, present in $\Omega_{i}$, the subdomain which contains the point with coordinates $(m, n)$. The error functional is now described by (note that because of the deterministic character of the model, the state is not estimated here):

$\Gamma(\underline{p})=\sum_{k=N_{1} j=1}^{N_{2}} \sum_{m}^{N_{m}} \alpha_{i}\left[\underline{Z}_{j}^{k}-\left(M \underline{Y}^{k}\right)_{j}\right]^{2}$

Now, by using simulated data from a two days period with a sampling time of a half hour, two important aspects of the calibration procedure can be analyzed. The first is the parameter estimation itself and second, the development of the standard deviations of the residuals $\left(\underline{Z}^{k}\right)_{j}-\left(M Y^{k}\right)_{j}, j=1, \ldots, N_{m}$ and $k=N_{1}, \ldots, N_{2}$.

The initial guesses for the bottom friction coefficients deviate largely from the true values and causes a root mean square error (averaged over $\Omega$ ) of $0.23[\mathrm{~m}]$ with extremes of $0.60-0.90[\mathrm{~m}]$. The effects of these deviations are reflected by incorrect magnitudes and circulation of the velocity fields in $\Omega$ and great phase shifts and amplitude errors of the water levels in the data observation points, see also the figures 7 and 8 . The results of the parameter estimation are shown in figure 4 . It demonstrates a remarkable difference between the estimation of $\lambda_{1}$ and $\lambda_{2}$ on the one hand and $\lambda_{3}$ and $\lambda_{4}$ on the other (an oscillating versus a monotonic behaviour): the influence of changes in $\lambda_{1}$ and $\lambda_{2}$ on $\Gamma(p)$ is far greater than changes in $\lambda_{3}$ and $\lambda_{4}$. This is caused by the reciprocal dependence of the bottom friction on the depth, so the friction in $\Omega_{3,4}$ is much smaller than in $\Omega_{1,2}$ and by the fact that the data observation points in $\Omega_{4}$ are suited close to the boundary which was assumed to be exactly known. Other reasons for the difference are the concentration of data observation points in the southern part of the North Sea, especially in $\Omega_{2}$, and the 


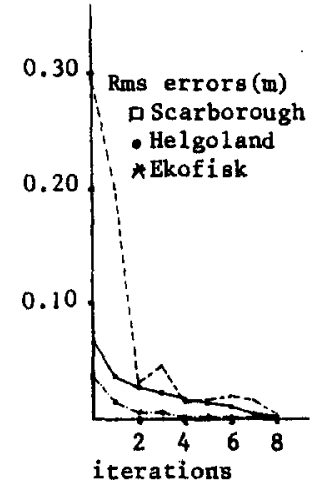

Figure 6

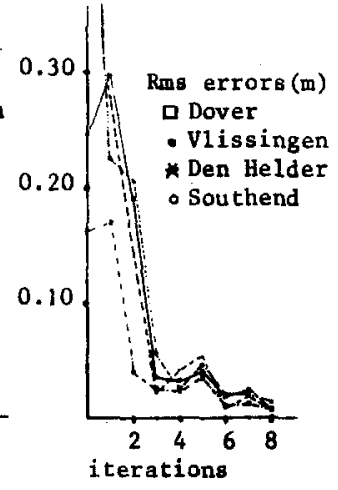

- V1issingen

* Den Helder
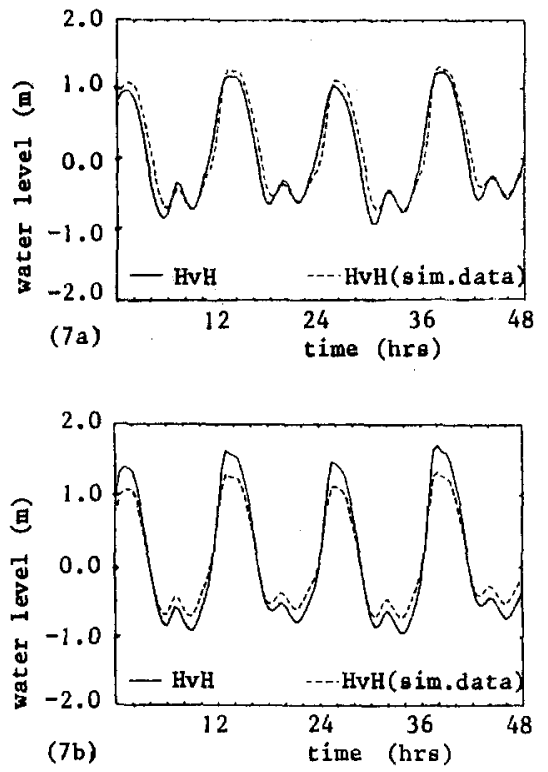

Figure 7

Figure 6. The r.m.s errors in the subdomains $\Omega_{2}$ and $\Omega_{3}$

Figure 7. The differences between the simulated data and (a) the water levels, calculated with the initial guesses for the bottom friction components; (b) the water levels, calculated with the bottom friction components after 1 iteration

non-linear interactions near the coast, due to local geometrical variations. Nevertheless, all the parameters are identified after about 8 iterations, within an accuracy of $2 \%$. After a closer analysis of the iterations, it can be shown that the algorithm has some difficulty in finding the correct second order properties of the error functional $\Gamma(p)$ for some components: it dependents too strong on the initial guess of the curvature, so if this initial guess is too large the parameter estimation converges to the wrong value. A last aspect of the iteration procedure that is worth mentioning is the way in which the r.m.s errors decrease. From the figures 7 and 8 it is clear that the effect of a phase shift is compensated first (after one iteration the phase shift is almost everywhere disappeared) whereas the correct amplitude is found after some more iteration steps with under- and overshoot.

\section{Parameter estimation in stochastic models}

As a first step towards the application of the estimation procedure to the stochastic models, we consider a domain with straight coast lines, see Fig. 9, where the dynamics are described by the linear equations (4)-(6) and the depth $D$ is constant over the domain $\Omega$. By prescribing an open boundary condition of the form: 

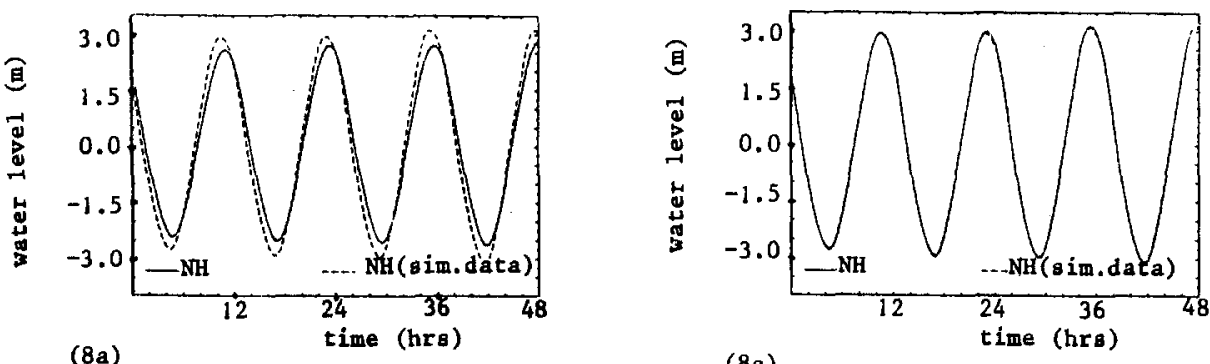

(8c)
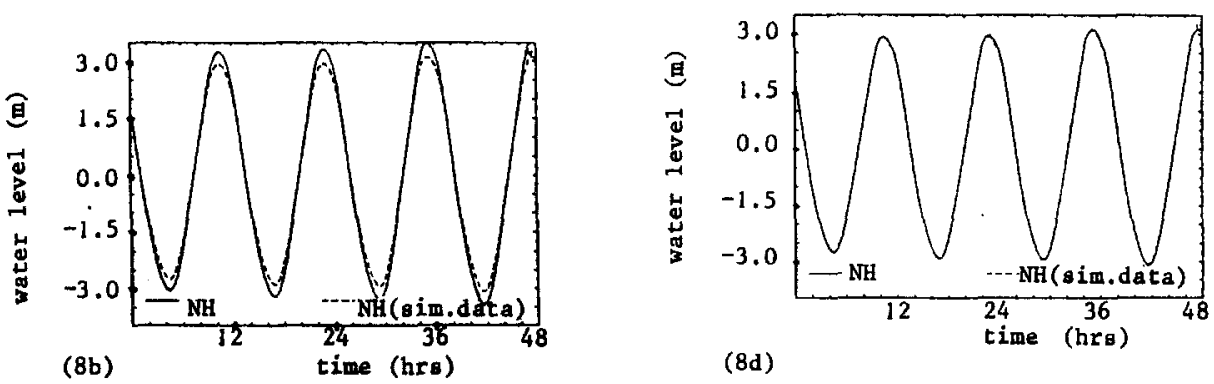

(8d)

Figure 8. The differences between the simulated data and the water levels, calculated with the bottom friction components after (a) 0 iterations (initial guesses); (b) 1 iteration; (c) 5 iterations; (d) 8 iterations

$v_{m, 0}^{k}=0.3 \sin \left(\omega k \Delta t+\phi_{m}\right), \quad \omega=0.000144\left[\mathrm{rads}^{-1}\right], \quad \phi_{m}=\frac{3 \pi}{16 x_{\max }}\left(x_{m}-x_{\max } / 2\right)$

at the northern boundary. Furthermore, the domain $\Omega$ is again divided into subdomains $\Omega_{i}, i=1,2,3,4$ where different bottom friction coefficients are introduced: $\lambda_{i}, i=1,2$, 3,4 . From this truth-model, a set of data is generated.

To represent an incorrect open boundary condition, in the simulation model a deterministic open boundary condition:

$v_{m, 0}^{k}=0.26 \sin \left(\omega t_{k}+\phi_{m}\right)+0.03 \sin \left(2 \omega t_{k}\right)$

is assumed. If the influence of this incorrect open boundary condition is not taken into account, the successive estimates of the bottom friction coefficients are used to compensate this error. A convergent series of estimates is found, but the interpretation of the results as physical coefficients is not possible. For instance, they can become negative. Moreover, it is not possible to decrease the value of $\Gamma(p)$ to a level that can be associated with the amount of (artificial) measurement noise that we introduced to generate simulated data, which is equal to $N \cdot N_{m} \sigma_{m}^{2}, \sigma_{m}^{2}=0.002$ being the covariance of the measurement error. It is therefore clear that, if one wants to interprete the estimates as some unknown model parameters, it is necessary to model that there are more sources of model errors. In our case this is done by following the derivation from section 2 and by introducing a stochastic process $q\left(x_{m}, t_{k}\right)$ in the open boundary condition: 


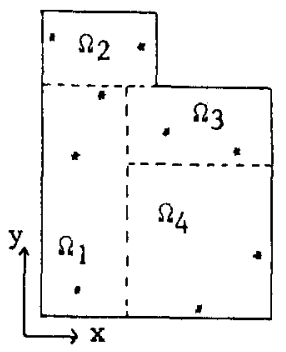

Figure 9. The geometry of the linear test model, used in section 7. The * denote the measurement points
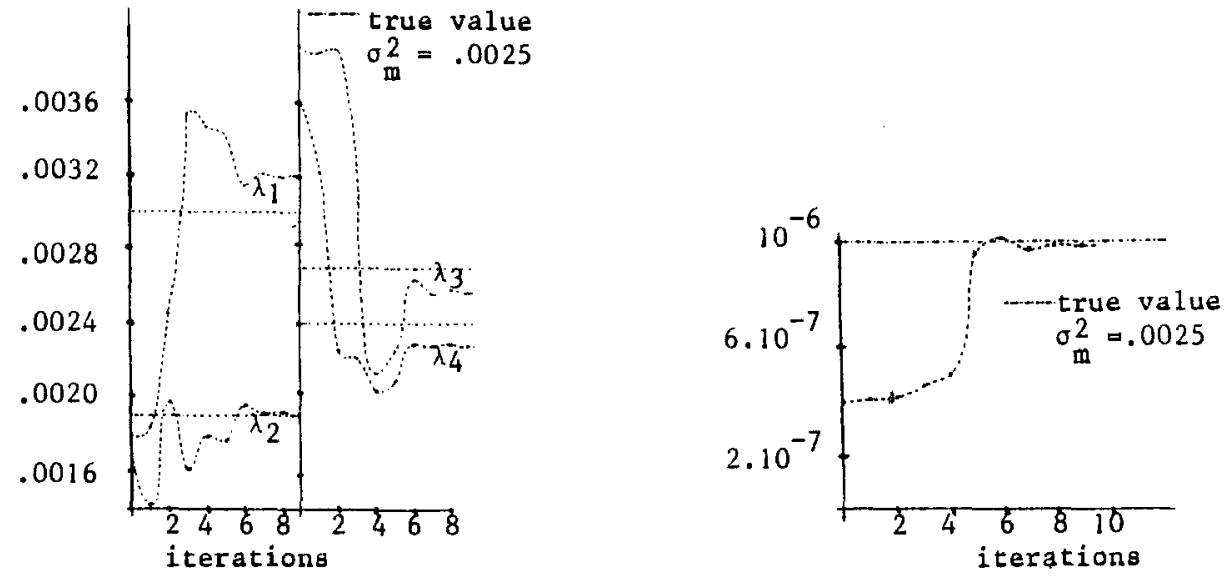

Figure 10

Figure 11

Figure 10. The estimation of the $\lambda_{i}, i=1,2,3,4$ in case the open boundary is uncertain, with $\lambda_{m, n}=F_{m, n}+\lambda_{i}$ and $\lambda_{m, n}$ defined in equations (15)-(20)

Figure 11. The estimation of $\gamma$ in case the open boundary is uncertain

$v_{m, 0}^{k}=0.26 \sin \left(\omega t_{k}+\phi\left(x_{m}\right)\right)+0.03 \sin \left(2 \omega t_{k}\right)+q\left(x_{m}, t_{k}\right)$

Because of the elementary nature of the example we assume that the spatial dependence of $q\left(x_{m}, t_{k}\right)$ is given by:

$q\left(x_{m}, t_{k}\right)=\mu_{m} q_{1}\left(t_{k}\right)+\left(1-\mu_{m}\right) q_{2}\left(t_{k}\right), \quad \mu_{m}=\frac{x_{\max }-x_{m}}{x_{\max }}$

The difference between the actual and the deterministic open boundary condition can be described by the processes $q_{1}\left(t_{k}\right)$ and $q_{2}\left(t_{k}\right)$ which evolve according to:

$q_{1,2}\left(t_{k+1}\right)=0.9 q_{1,2}\left(t_{k}\right)+\eta_{1,2}, \quad \eta_{1,2}=N\left(0, \sigma_{1,2}^{2}\right)$

where $\eta_{1}$ and $\eta_{2}$ are white noise processes. A consequence of the introduction of $q_{1}\left(t_{k}\right)$ and $q_{2}\left(t_{k}\right)$ is that the state vector has to be redefined: 
$\underline{Y}^{k}=\left[\underline{X}^{k}, q_{1}^{k}, q_{2}^{k}\right]^{T}$

and a system- $(=\underline{W})$ and measurement noise sequence $(=\underline{V})$ have to be introduced:

$\underline{\tilde{W}}=\left[\underline{0}, \eta_{1}, \eta_{2}\right]^{T}$

to derive the stochastic system representation:

$\underline{Y}^{k+1}=\Psi \underline{Y}^{k}+\underline{\tilde{W}} \quad ; \quad \underline{Z}^{k+1}=M \underline{Y}^{k}+\underline{V}$

In this model two types of errors are present: errors in the boundary condition and unknown parameter values. However, the way to treat these errors is not the same because the parameters are assumed to be time invariant, whereas the new states $q_{1}\left(t_{k}\right)$ and $q_{2}\left(t_{k}\right)$ are time dependent. The proposed procedure to calibrate the model assumes that the effects of these two sources of error are relatively independent, which implies that the corrections due to these two types of errors can be calculated separately. Now, the system representation, equations (57)-(58), is a linear one with time independent noise sequences. The steady state Kalman filter can therefore be used to estimate the state $Y^{k}$ (thus also estimating $q_{1}\left(t_{k}\right)$ and $\left.q_{2}\left(t_{k}\right)\right)$ by processing the data and correcting the state for the inaccuracies due to the errors in the open boundary condition. The effects of the other source of error are compensated by finding suitable values for the parameters which minimize the error criterion:

$\left.\min _{\underline{p}} \Gamma \underline{p}\right) ; \quad \Gamma(\underline{p})=\sum_{k=1}^{N}\left\|\underline{Z}^{k}-H \underline{Y}(k \mid k, \underline{p})\right\|^{2}$

The method that is used here to find the minimum of $\Gamma(p)$ with $p$ the parameter vector, consisting of 4 bottom friction coefficients and one (global) wind stress coefficient, is described in section 5 . The results of the combined filtering and estimation procedure are shown in the figures 10 and 11 . It is obvious that, although the percentual error in the prescription of the open boundary condition is about $23 \%$, the differences that remain between the estimations of the parameters and the true values are relatively small.

\section{Conclusions and remarks}

After the proposed estimation procedure is explained and illustrated with some examples, we can summarize the results as follows:

- the parameters, the friction coefficients as well as the wind stress coefficient, are reconstructed within a good accuracy. The remaining difference between the final values of the estimates and the true values are only small, whereas the minimal value of the criterion $\Gamma(p)$ apprximates the value that is related to the total amount of measurement noise, the simulated data are disturbed with. This point applies both for the cases with and without uncertainties in the open boundary prescription.

- The number of iterations that is required to ensure the iteration process to converge is approximately (linear test model) - 8 (CSM-16 model). In the case where the wind stress coefficient $\gamma$ was not simultaneously estimated in the linear test model it was even less. As already mentioned in the section about the estimation algorithm, the BFGS-method converges in $(N+1)$ steps if $N$ is the number of parameters to be estimated, the cost function $\Gamma(p)$ is purely quadratic and the data are noise free. So, if the number of iterations here is 6-7 and the number of parameters that are estimated simultaneously is 5 , we may conclude that the cost function is nearly quadratic with respect to $\lambda$ and/or $\gamma$. 
- The acceleration that can be found by replacing a first order method (where the direction of search is the opposite of the gradient $\nabla \Gamma(p)$ ) through a quasi second order one is quite huge, in our case it has turned out to be a factor 5.

- The results that are shown in section 7 shows the possibility of the filter to compensate for errors in the open boundary condition: the effect of the state estimation is clear. The estimation procedure assumes the relatively independence of the errors in the states that were due to the incorrect boundary condition and the errors due to the unknown parameters. From experience we conclude that this assumption holds.

- In the introduction we mentioned that a total calibration procedure also included the adjustment of the geometry. One of the straightforward possibilities to include this aspect in the estimation procedure is to divide the domain $\Omega$ into subdomains (which do not necessarily have to coincide with the subdomains for the bottom friction) and to represent the depth in a point with coordinates $(m, n)$ as $D_{m, n}=D_{m, n}^{g}+$ $D_{i}$ where $D_{m, n}^{g}$ is given at forehand and $D_{i}$ is a quantity to be estimated and which is constant over the newly introduced subdomains.

\section{Acknowledgement}

The authors gratefully acknowledge the stimulating discussions with Prof. A. Bagchi, Dr. B. de Jong, Mr. J.G. de Ronde and Mr. A. Langerak.

\section{References}

Budgell, W.P. 1981: A stochastic deterministic model for estimating tides in branched estuaries. Report No. 10, Ocean Science and Surveys, Fisheries and Oceans, Burlington

Chiu, C.L. (ed.) 1978: Applications of Kalman filter theory to hydrology, hydraulics and water resources. Univ, of Pittsburg, Pittsburg

Chavent, G. 1979: Identification of distributed parameter systems: about the least square method, its implementation and identifiability. Proceedings 5th IFAC Symposium on Identification and System Parameter Estimation, Pergamon Press, Darmastadt

Dronkers, J.J. 1964: Tidal computations in rivers and coastal waters. North Holland Publishing Co., Amsterdam.

Fletcher, R. 1980: Practical methods of optimization. Vol. 1 \& 2, Wiley-Interscience Publication

Heemink, A.W. 1988: Two-dimensional shallow water flow identification. Applied Mat. Mod., 109-118

Heemink, A.W.; ten Brummelhuis P.G.J. 1989: Open channel flow identification: case studies. Proceedings of the Symposium on Open Channel and Runoff Catchment (to appear)

Jazwinski, A.H. 1970: Stochastic processes and filtering theory. Academic press

$\mathrm{N}$ Kaoua, $\mathrm{T}$.: Calage de modele, identification du strickler dans les equations de saint venant. Electricite' de France, Direction des Etudes et Recherches

Morf, M.; Sidhu, S.S.; Kailath, T. 1974: Some new algorithms for recursive estimation in constant, linear, discrete time systems. IEEE Trans. Autom. Control, AC-19, 315-323

Safonov, M.G.; Athans M. 1978: Robustness and computational aspects of non-linear stochastic estimators and regulators. IEEE Trans. Autom. C., AC-23

Stelling, G.S. 1983: On the construction of computational methods for shallow water flow problems. Rijkswaterstaat Communications, The Hague, 35.

ten Brummelhuis, P.G.J.; Jong, de B; Heemink A.W. 1988: A stochastic dynamic approach to predict water-levels in estuaries. Journ. of Hydr. Engin. 114, 1339-1358

Verboom, G.K.; van Dijk, R.P.; Ronde, de J.G. 1987: Een model van het Europese continentale plat voor windopzet en waterkwalieitsberekeningen, RWS-DGW/WL, GWAO-87.021, (in Dutch).

Accepted May 30, 1990 